\title{
Eye Gazes Based on Associative Relevance Assist in Decision Making Processes during Scene Perception
}

\author{
Gufran Ahmad $^{1}$ \\ ${ }^{1}$ College of Computer Sciences and Information Systems, Jazan University, Jazan, Saudi Arabia \\ Correspondence: Gufran Ahmad, College of Computer Sciences and Information Systems, Jazan University, \\ Jazan, Saudi Arabia. Tel: 966-503-082-048. E-mail: gufran.researcher@gmail.com
}

Received: January 14, 2016

Accepted: February 8, 2016 Online Published: March 25, 2016

doi:10.5539/ijms.v8n2p74

URL: http://dx.doi.org/10.5539/ijms.v8n2p74

\begin{abstract}
Research studies on eye movements in area of information processing task, such as scene perception have recently advanced towards understandings of underlying visual perception mechanism and human cognitive dynamics. Besides, business applications of eye tracking are endlessly revealing groundbreaking trends based on practical scenarios. In this study, we conducted a number of eye tracking experiments to establish our hypothesis that the eye gazes based on the associative relevance found within the contexts of scenes during scene perception significantly supported the processes of decision making. The collected eye movement data from participants who viewed artistic scenes discovered that the tracks of eye gazes traversed along the existing associative relevance among the elements of scenes for decision making processes. These experimental evidences confirmed our hypothesis that the eye gazes based on associative relevance assisted in decision making processes during scene perception.
\end{abstract}

Keywords: decision making, eye gazes, associative relevance, scene perception

\section{Introduction}

\subsection{Introduction and Importance}

Studies on eye movements are undergoing in the advanced phase of innovative approaches and solutions. A number of interdisciplinary demands and interests have extended eye movement researches into numerous perspectives of social, economic, business, and scientific scenarios. Research areas like, human and developmental psychology, psycholinguistic and readings, neuroscience, vision research, usability studies, business marketing and advertising research, ophthalmology, human computer interaction are trending for more profound interests than ever before and eye movement studies are taking part in these areas. Additionally, these studies have improved research visions and insights, tools and techniques, and extensive applications. Tracking of eye movements leads to noteworthy comprehensions of human mind, in terms of human intentions that are the strategic concerns for businesses because businesses can make acquainted with human conducts, and attitudes to make business plans and policies accordingly. Further, customers' opinions and purposes are necessities for better business and market behavior that mainly depends on their behaviors. Currently, a number of online and offline businesses, like marketing, advertisement, shopping, search engine optimization, and web designing are converting and revolutionizing their business strategies and objectives in accordance with the innovative outcomes of eye movements for business growths (Granka, Joachims, \& Gay, 2004; Bojko, 2013; Koller, Salzberger, Brenner, \& Walla, 2012; Pan, Hembrooke, Gay, Granka, Feusner, \& Newman, 2004; Hermansen, 2015; Pan, Hembrooke, Joachims, Lorigo, Gay, \& Granka, 2007; Ahmad, 2015).

Usually, eye movements involve in the verbal and nonverbal communications and assist in providing the desired information among the participants of communication. In addition to these, eye movements actively contribute in the processing of data for information and the visualization of information that are common practices among professionals and individuals, including artists and scientists. In reality, this complex mechanism of neurocognition is a combination of numerous underlying processes existing in human mind. The human mind incites and causes cognitive processes, like human's sensation, consciousness, visual attention, perception, meta-cognition, reasoning, analogical thoughts, information processing, and other concerned processes (Wells, 2000; Tsotsos, 2011; Tommasi, Peterson, \& Nadal, 2009; Stark \& Ellis, 1981; Snowden, Thompson, \& Troscianko, 2012; Holsanova, 2008; Rayner, 1992; Bly, 1999; Ahmad, 2011; Ahmad, 2013; Ahmad, 2014; 
Ahmad, 2015).

In eye movement processes, eyes change their gazes to spot a specific portion of the visible region in viewing because of having tendency to perceive the degree of detail visible in the central direction of gaze. In the movements, they pass through two temporal phases: fixations (the stops or periods of time when point of gaze or significant look is relatively slow) and saccades (the hops between stopping points). Saccades are often information seeking and directed to specific objects or regions by the requirements of ongoing behavior. This infers the existence of cognitive processes of eye movements in viewing as well. Therefore, the underlying mechanism of visual viewing is sequential and coordinated phenomena of cognitive as well as correlated processes (Bojko, 2013; Brown, 1999; Duchowski, 2003; Gompel, Fischer, Murray, \& Hill, 2007; Henderson \& Hollingworth, 1998; Henderson \& Hollingworth, 1999; Hoffman, 1998; Holsanova, 2008; Liversedge, Gilchrist, \& Everling, 2011; Rayner, 1992; Snowden, Thompson, \& Troscianko, 2012; Stark \& Ellis, 1981; Ahmad, 2011; Ahmad, 2014; Ahmad, 2015).

\subsection{Related Background and Corresponding Research Design}

The study of relationships of fixation sequences of eye movements to the behavior of usual human activities has its origin. In fact, inherent salience of objects is not accountable for focal shifts in eye movements, but by their importance to the task or context in hand. In contradiction of free viewing, the movements of eyes and contextual actions are associative in nature and have a chain of linking. Moreover, contextual actions compose of a number of perspectives including an act of associativity within the contexts, elements, or intents. Such associativity has built upon the sense of relevance among the contexts of the object (Stark \& Ellis, 1981; Snowden, Thompson \& Troscianko, 2012; Rayner, 1992; Nikolaev, Pannasch, Ito, \& Belopolsky, 2014; Liversedge, Gilchrist, \& Everling, 2011; Holsanova, 2008; Hoffman, 1998; Henderson \& Hollingworth, 1999; Henderson \& Hollingworth, 1998; Griffin, 2004; Gompel, Fischer, Murray, \& Hill, 2007; Ahmad, 2014; Ahmad, 2015).

In addition, a focused visual appearance, i.e., spotlight metaphor, enables and constructs the visual focus of attention in eye movements during artistic scene viewing. The center of focused attention in visual area is considered as having more brightness than areas to which attention is not focused or areas from which attention has been detached. The spotlight of attention switches off at one location and then switches on at other. Besides, as human's capabilities for attention and processing for information has restraints cognitively, so it is rather challenging to consider about focusing everything at a time. Cognitively, human gives attention to small part of visual object, one at a time and likewise, human can focus on only small piece of information at a time. This is why the human processing of information is reasonably gradual as it works on the mechanism of human cognition (Bly \& Rumelhart, 1999; Gompel, Fischer, Murray, \& Hill, 2007; Griffin, 2004; Hoffman, 1998; Holsanova, 2008; Liversedge, Gilchrist, \& Everling, 2011; Nikolaev, Pannasch, Ito, \& Belopolsky, 2014; Snowden, Thompson, \& Troscianko, 2012; Stark \& Ellis, 1981; Ahmad, 2014; Ahmad, 2015).

As a further matter, there occur numerous impact factors, which engender reasoning, notion of analogy, flow of thoughts, meta-cognition, and other pertinent cognitive processes during the eye movements. We regard in a location of scene that is partly determined by the scene's constraints and region's informative description, partly by the task, intent, context, or interest. Viewers can arrange diverse visual paths through the same scene, since they extract information from those parts of the scene to describe particularly. Therefore, the evolved flow of thoughts cognitively, motivates the conscious focus of attention to change to the next contextual part of the scene. By doing so, these impacting factors propel human visual focus of attention dynamically in the phenomena of eye movements (Wells, 2000; Tsotsos, 2011; Stark \& Ellis, 1981; Rayner, 1992; Liversedge, Gilchrist, \& Everling, 2011; Holsanova, 2008; Hoffman, 1998; Henderson \& Hollingworth, 1999; Ahmad, 2014; Ahmad, 2015).

Normally, an art is a human way of cognitive activities and the art has the purpose to influence the minds of people who looked at them. Art is productive activity that focuses on the thoughtful modification and embellishment of worldviews. Mostly, all known pieces of art are creative and metacognitive as per their roles because they are self-explanatory. The types and styles of art are technology-driven as innovative technologies bring renaissance to the artworks. The most essential part of art is its motive to become conscious about itself and in turn, firing up the cognitive processes in human mind. In addition, as science is laying its foundation to understand our knowledge about art, likewise, the art offers us a view of mind that understands the art. Undoubtedly, we identify that all types of art are one of the fabulous representations in our lifetimes. It can calm down our distressed heart and motivate our mental states and spirits. Further, artistic perceptions stimulate profound thoughts as well as all types of sensations. Along with other sensations and perceptions, an art 
stimulates the human emotions as well. That is an integral part of human intelligence, so that the onlookers of artworks may sense and perceive a novel lookout (Holsanova, 2008; Solso, 1994; Solso, 2003; Turner, 2006; Viegas \& Wattenberg, 2007; Ahmad, 2011; Ahmad, 2013; Ahmad, 2014; Ahmad, 2015).

During artistic scene observation, we move our eyes rapidly in irregular manner to change focus from one fixation to another fixation. This process, saccade, is one of the most common behavior of eyes. Pattern is obtained only during the periods of relative gaze constancy, known as fixations. The process of directing the eyes to view picture in real time is known as gazing of eyes. The processes of eye movements bring about and propagate a series of streaming thoughts successively to obtain information about the scene or object of interest. These streams of thoughts predominantly originate the notion of analogy. Although there are a number of standpoints about analogy itself, yet analogical impression is constantly accessible in visual perceptions. Based on existing research works, it gives the consideration that the standpoint of mapping and the standpoint of higher level of perception are two dissimilar looks of the same thing, i.e., analogy. Analogy is one of the inherent concepts that persist during the phenomena of eye movements, although it is not the complete reason to believe as this does not explain the whole scenario (Wells, 2000; Viegas \& Wattenberg, 2007; Turner, 2006; Tsotsos, 2011; Solso, 2003; Solso, 1994; Holsanova, 2008; Hofstadter \& Sander, 2013; Gentner, Rattermann, \& Forbus, 1993; Gentner \& Medina, 1998; Gentner \& Markman, 1997; Gentner, 1983).

There comes the process of associative relevance that is progressive and coexisting cognitive processes and are emanated from thoughts of analogy and continue to flow during entire timespan of visual scene viewing. These generated processes of associative relevance bring about the process of associations among the relevant entities or contexts. These associative relevance phenomena pass through human cognitive process as an aid during scene viewing, take part in focal shifts of eyes during active scene viewing as well as facilitate in decision making process by choosing the optimal route of alternative. Associative relevance is developmental and interrelated notion, which originates from thoughts of analogy during the process of human viewing. The influence of associative relevance predominantly remains during cognitive phenomena of eye movements. It is an underlying mechanism for creativity and annihilation of complexity during information processing and information retrieval for better interpretation of the object of interests. Besides, it resembles as an associative chain that links contexts, intents, portions, elements, shapes, sizes, colors, contrasts, or relations based on similarity or sameness. Further, associative relevance manipulates and generates an incitement cognitively to associate relative contexts, intents, elements, etc. The process of associative relevance initiates during the shifts in visual focus of attention as the movements of eyes establish associations among analogical contexts or portions of interest during the active scene viewing (Gentner, 1983; Gentner \& Markman, 1997; Gentner \& Medina, 1998; Gentner, Rattermann, \& Forbus, 1993; Gompel, Fischer, Murray, \& Hill, 2007; Holsanova, 2008; Ahmad, 2014; Ahmad, 2015).

\subsection{Decision Making Process and Proposed Hypothesis}

Sited literatures (Stark \& Ellis, 1981; Rayner, 1992; Liversedge, Gilchrist, \& Everling, 2011; Holsanova, 2008; Hoffman, 1998; Henderson \& Hollingworth, 1998; Griffin, 2004; Gompel, Fischer, Murray, \& Hill, 2007) suggest that human eye saccades are information seeking in nature and are engaged in the job of retrieving information. However, the gazes play significant role, as they get involved in decision making, so that new information based on the previous decision might be sought. Further, the decision might be verified for future purposes as well. Besides, the eye gazes act as junctions that undergo through the processes of decision-making under the associated cognitive processes of influential nature (Adair, 2010; Bird, Lauwereyns, \& Crawford, 2012; Nikolaev, Pannasch, Ito, \& Belopolsky, 2014; Orquin \& Loose, 2013; VanRullen \& Thorpe, 2001).

The movements of eyes consisting of gazes and saccades have a clearly defined objective that may be organized in several alternative ways of movements by making a decision based upon choosing from a set of possible alternatives under the guidance of the cognitive processes and analogical thoughts. Each choice offers its own advantages and disadvantages, so that in a complex situation, the decision maker might not be able to make a preferable option at once and quickly decide why he or she should prefer one alternative and not another. To clarify the situation and compare the alternatives in several aspects, inherent human information processing along with associative relevance phenomena suggests a series of cognitive operations. Their aims are to analyze the situation critically and thus prepare a decision to move ahead during active scene viewing phenomena (Adair, 2010; Bird, Lauwereyns, \& Crawford, 2012; Holsanova, 2008; Nikolaev, Pannasch, Ito, \& Belopolsky, 2014; Orquin \& Loose, 2013; VanRullen \& Thorpe, 2001).

We propose the hypothesis that associative relevance based human eye gazes assist in decision making processes during the scene perception. This proposed hypothesis is completely based on cognitively generated underlying 
mechanism consisting of indivisible flow of thoughts that produces the process of associative relevance profoundly.

An undertaking (a system of actions initiated by eye gazes) having a clearly defined objective may be organized in several alternative ways (eye gazes) of fixations by making a decision based upon choosing from a set of possible alternatives produced by series of fixations. Each choice offers its own advantages and disadvantages, so that in a complex situation, the decision maker might not be able to make a preferable option at once and quickly decide why human should prefer one alternative and not another. To clarify the situation and compare the alternatives in several aspects, eye movements suggest a series of operations based on cognitively evolved associative relevance process. Their aim is to analyze the situation critically and thus prepare a decision for those bearing the responsibility for a final choice by decisive path of gazes based on associative relevance that ultimately assists in making decision within the contexts of visual scenario.

\section{Method}

We investigate the gazing of eye movements from cognitive perspective, including the associative relevance process, during scene viewing and analyze the patterns of sequenced gazing to visualize the information. Here, visualization of sequenced gazing patterns to extract information for interpretation is essential steps of this study.

Initially, eye movements, in terms of sequenced gaze, are collected from participants who view full-color scenes while engaging in a visual search task in which they are freely viewing different fields of each scene. Finally, we compare and analyze the sequenced gazing against the artistic scene. The interpretation is carried out with the help of cognitive and analogical processes in current research.

\subsection{Eye Tracking System}

In eye tracking system, the system illuminates infrared light for tracking the eye movements. The camera, connected to the system, captures the location of viewer's eyes in terms of fixation during experimentation time. As the viewer moves his/her eyes to look a new location of the scene, the camera records new fixation also. This process of recording continues subsequently. The system generates eye movement tracks and heat maps using the captured data which is utilized for further analysis.

The schematic diagram of eye tracking system and basic processes involved during eye tracking experimentation is represented in Figure 1.

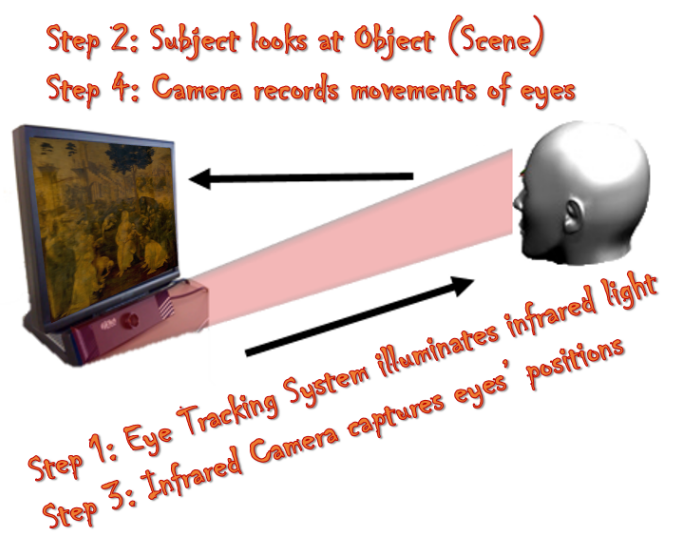

Figure 1. Eye tracking system with operational processes

The traces of eye movements are taken in diverse layouts as per analyst's suitability. Among them, there are two most common formats are Heat Map and Sequenced Gazing with circle of concentration. In Heat Map, the track of eye is recorded as illumination and intensity of infrared light rays. This is based on Energy Therapy Technique (ETT). In Sequenced Gazing, the eye tracks are entered as numbered circles with their areas indicating the time duration of eye's gazing in those areas respectively (Bojko, 2013; Brown, 1999; Duchowski, 2003; Holsanova, 2008).

In our experiments, we study track of eye fixations as the sequenced gazing of viewer's eye movements, which is generated by the system, during scene viewing. These are the dynamic shifts of eye gaze in scene viewing. 


\subsection{Flow Chart of Study}

The study on eye movements during scene viewing consists of a number of steps to be performed. These steps are represented as shown in the adjacent flow chart diagram (Figure 2). This is a comparative study of two items; one item is artistic scene and other item is the eye movement tracks of the same scene, which is generated from eye tracking system.

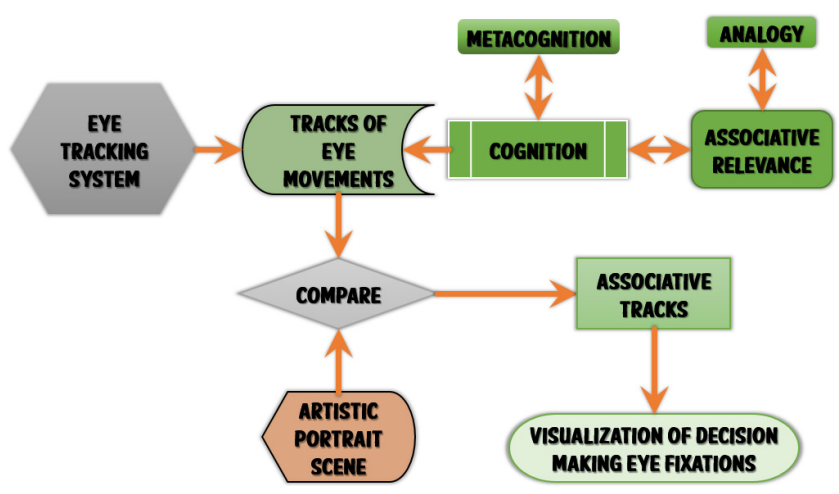

Figure 2. Flow chart of research study

It begins with recording of eye movement tracks for a Subject; a viewer on eye tracking system for an Object; artistic scene. The generated eye movement tracks of the same artistic scene are comparable to the original artistic scene. This comparative analysis infers visualization and interpretation of the outcome. So, we compare these two items side by side.

During comparison stage, we make use of cognitive process including metacognitive process, in addition to analogy based associative relevance mechanism that is happening consistently. These underlying processes generate crucial correlation that creates resultant maps. By analyzing, we come up with concluding remarks about the dependency of eye gazes on the associative relevance that controls and enhances the process of decision making in human mind.

\subsection{Experimental Procedure}

We selected 64 participants from a number of fields randomly, aging from 19 years to 42 years. Further, we assigned these participants (Subjects) to view two randomly selected famous artistic scenes (Objects) as shown below in Figure 3.

The artistic scenes were "Tuscan landscape in the Arno valley", and "The adoration of the Mary for the monks".
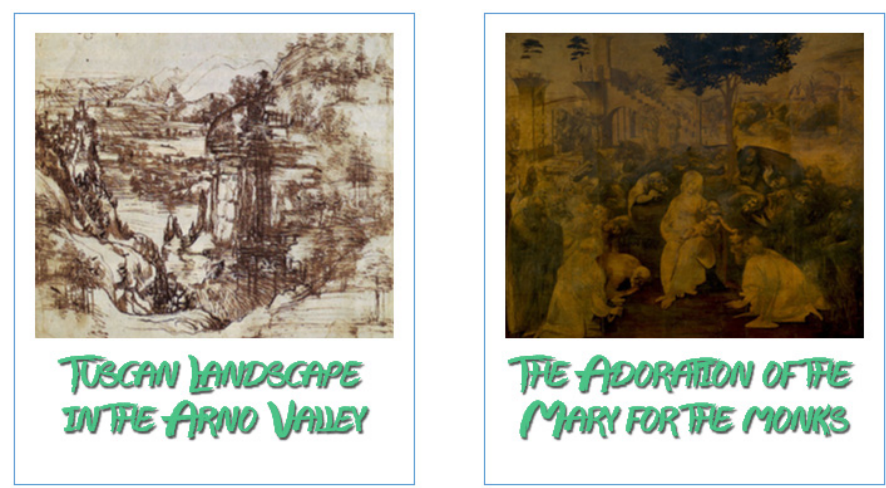

Figure 3. Selected artistic scenes for research study

Their eye movements were closely monitored as they viewed 32 bits full-color artistic scenes. The Objects, the scenes were displayed on a computer monitor. The scenes were shown at a resolution of $1280 \times 1024$ pixels and subtended $15 \mathrm{deg}$. horizontally by $10 \mathrm{deg}$. vertically at a viewing distance of $75 \mathrm{~cm}$. Eye position was sampled from an Eye Tech Digital Systems TM3 16 mm Eye Tracker, and eye tracking data was parsed into sequenced 
gazing with circles of concentration.

The Subjects' heads were held steady in advance prior to experimentation. Prior to the first trial, Subjects completed a procedure to calibrate the output of the eye tracker against spatial positions on the display screen. This procedure was repeated regularly throughout the experiment to maintain high level of accuracy. Subjects were initiated to view the scenes freely.

The scenes were presented to the Subjects for maximum duration of 60 seconds. During this time span, the Subjects viewed the scenes with their normal eyes and focused attention on the Object, the scene.

\section{Analysis}

In this phase of analysis, we analyzed the two scenes respectively with the intention to elaborate our findings in the most common and generalized perspective.

\subsection{Study of Artistic Scenery "Tuscan Landscape in the Arno Valley"}
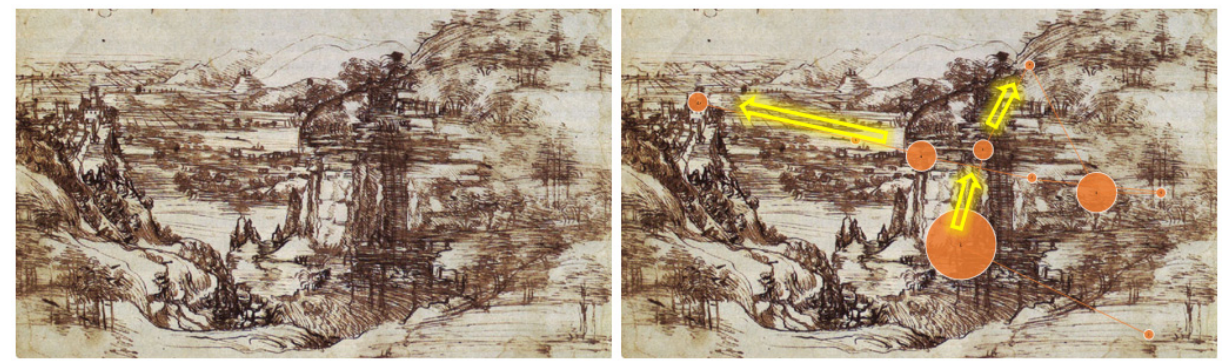

Figure 4. Artistic scenery of Tuscan landscape in the Arno valley and eye movement tracks of the scenery

In the experimentations of Figure 4, we tracked the eye movements for the artistic scenery of Tuscan landscape in the Arno Valley by Leonardo da Vinci.

In the beginning, the Subjects gazed their eyes towards the bottom right side of the scene. Next, they moved their eye gazes towards the center of the scene where sensation and consciousness perceived visual attention for visual perception. The Subjects viewed all of the visible area downward of the mountain. There were a number of trees along the sloppy hill-side road. At this moment, the Subjects' minds passed through analogical thought that cognitively evolved associative relevance process for creating associations among the relevant contexts of the scene, in terms of the relevant portions of the scene. At the same time, driven by the process of associative relevance within the context, the Subjects decisively moved to the next eye gaze in the track. Subsequently by doing so, they gazed their eyes at the upper portion of the scene. This ensured that the process of associative relevance acted as stimulus to propel the eye gazes for the next destination, and in turn, the same process of associative relevance assisted the eye gazes in making a decision to move along.

Then, by losing the process of previously created associative relevance due to the lack of associative context in the element of the scene, eye gazes returned downward for seeking information and processing of that information. However, the eye gazes could not perceive any contextual information that could establish associative relevancy from the previous known process of associative relevance. Finally, the eye gazes returned back to the sloppy hill-side road and moved away towards the left side of the scene for seeking information and the processing of the same information based on previous existing associative relevance mechanism. At this stage, it turned out that the existing associative relevance based on the contextual relevancies in the portions of scene had contributed in the entire scenario and acted as an assist for decision making processes to happen within the Subjects' minds cognitively.

Later, the entire cognitive mechanism of the scenario was confirmed by the Subjects' feedbacks in terms of questionnaires. Hence, we concluded that the eye gazes based on associative relevance assisted in decision making processes during scene perception. 


\subsection{Study of Artistic Scenery "The Adoration of the Mary for the Monks of San Donato a Scopeto"}
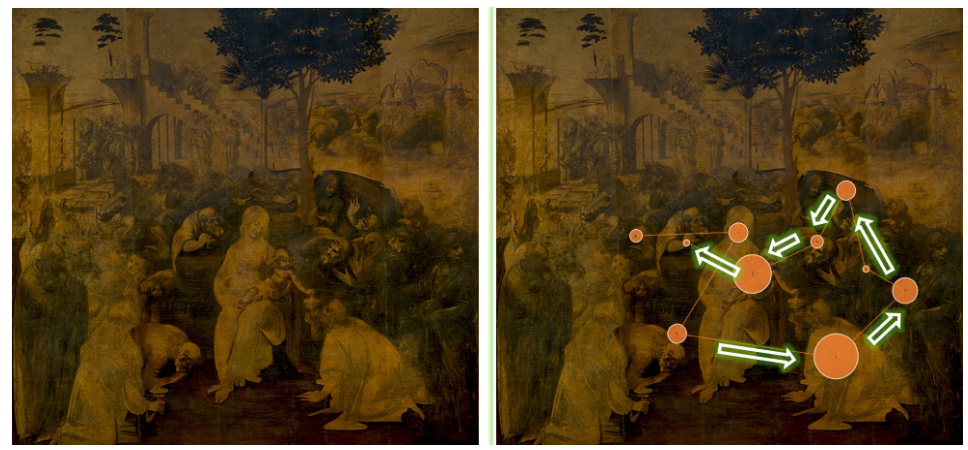

Figure 5. Artistic scenery of the adoration of the Mary for the monks and eye movement tracks of the scenery

In experimentations of Figure 5, the artistic scenery of adoration of the Mary for the monks illustrated the event of holy Child Jesus and the mother Mary sitting under a tree and surrounded by praising monks for respect and worship.

Initially, the Subjects gazed at the central left portion of the scene and gradually, moved towards the central portion where the facial expression of mother Mary was traced. Subsequently, the eye cases moved to the downward portion of the scene came across the leaned monk showing facial expression and hand gesture, and trying to kiss the holy feet. At this moment, the inherent human emotions motivated and initiated the process of human cognition. Due to the sensation and consciousness, visual perception was established along with the thought of analogy that proceeded in the next move of eye gaze towards the monk emotionally sitting on the ground with respect. Existence of such thought of analogy cognitively evolved the process of associative relevance that formed associative chaining of the relevant contexts built up the human emotions as reflected in facial expressions and hand gestures within the elements of the scenery.

In the next move, the eye gaze shifted at the hand gestures of adjacent monk due to the same associative relevance process having involvement of human emotion. Subsequently, the eye gaze further moved towards the upper portion of the scene for seeking information based on the same analogy and established associative relevance among the related contextual elements within the scenery. Once again, the hand gestures and facial expressions founded the continuous process of associative relevance. In the next successive moves, the eye gazes were directed and driven by the same contextual elements of human emotions found within the similar portions of scenery. Further, we revealed that the underlying processes of associative relevance formed a basis for the processes of decision making in Subjects' minds during the scene viewing, so that the eye gazes moved accordingly and decisively towards the proper direction (as guided and instructed by the process of associative relevance) within the portions of the scene.

Later, the cognitive processes and involved mechanism happened within the eye tracking scenario received a definite confirmation by the Subjects' feedbacks in their responses of assigned questionnaires. Therefore, we concluded that the eye gazes based on associative relevance assisted in decision making processes during scene perception.

\section{Discussion}

In this research work, we choose some of the finest pieces of art intentionally. The artistic sceneries embrace in their manifestation with the foremost purpose of artistic artworks in the shape of human cognition mechanism in viewing these artistic sceneries. These creative pieces of art reflect inherent human interaction to perceive knowledge and interpretation of realistic world in human mind. These emotional views are rather too complicated to understand from visual analytics and analytical reasoning. Consequently, these cognitive perspectives, i.e. thinking of analogy and process of associative relevance are discovered by the tracks of eye gazes in scene viewing (Wells, 2000; Viegas \& Wattenberg, 2007; Turner, 2006; Solso, 2003; Solso, 1994; Liversedge, Gilchrist, \& Everling, 2011; Holsanova, 2008; Hofstadter \& Sander, 2013; Ahmad, 2011; Ahmad, 2013; Ahmad, 2014; Ahmad, 2015).

Decision making has become a science rather than an art. Although in some of the management sectors, people still consider it as an art, yet the studies of decision making processes have revealed it as a science. In a number of cited literatures (Adair, 2010; Bird, Lauwereyns, \& Crawford, 2012; Holsanova, 2008; Nikolaev, Pannasch, 
Ito, \& Belopolsky, 2014; Orquin \& Loose, 2013; VanRullen \& Thorpe, 2001), the decision-making process is closely connected with the human cognition as there is a significant amount of contribution and control carried out by the human interaction. Moreover, most of the times, the decisions are made by the human within human-centric processes and scenarios.

Coexisting associative relevance processes from the flow of analogical thoughts during scene viewing is significant step for appropriate retrieval of task-relevant visual information which are essential for visualization of final maps. In this study, we observe that the generated eye movement tracks of gazes are strongly following the same path as directed and decided by the associative relevance processes. These facts show a clear tendency for decision making process to get initiation from the associative relevance based eye gazes. Therefore, the eye movements get manipulated operation under the guidance of associative relevance broadcast. As a result, without taking into account the influential factor; associative relevance propagation, it is impossible to link the entire scenario of human cognition in the sequential eye gazes of eye movement tracks.

The visualization of streaming associative relevance process in terms of followed eye movement tracks is a tactical and decisive part of whole activities. The visualization of decisive and controlled eye movement tracks, in terms of associative relevance, is unarguably innovative perspective of each and every analyst who examines them for definite intents of decision making processes. This, in turn, causes a number of associative relevance scenarios in scene viewing by various perspectives of analysts.

The directional shifts in eye gazes have correlated consequences along with the decision making processes that is happening cognitively within human mind and is consistently processing information together with the associative relevance processes. The visualization of relevant eye movement tracks reinforces again the existence of dominating associative relevance factor; the controller of entire phenomena in between the inherent cognitive and metacognitive processes during scene viewing.

In addition to these arguments, the experimental evidence of associative traversal path generated by eye tracks as the happening of decision making processes during active scene viewing holds our hypothesis for which we conducted a series of experimentations. The hypothesis that the associative relevance based eye gazes assist in decision making processes during scene perception, is persuasive and pioneering breakthrough related to eye movements study.

\section{Future Research Plan}

Thus far, the current research has approached towards better understanding of dynamics of human cognition during visual perception from the perspective of the decision making process in human mind within the framework of involved underlying mechanism of visual attention and perception along with mandatory process of associative relevance.

The proposed model and experimentations are satisfactory but need more revival for the much highly considerations. Our future task includes finding more robust and detailed model for the explanations of current phenomena of eye movements during advanced scenarios.

Additionally, one of the future plans for the current research findings comprises finding additional influential factors during eye movements within contextual states and systems of research interest.

\section{Acknowledgments}

At first, our sincere and earnest thanks go to the participants who donated their precious time and efforts during the entire experimentations. We are indebted to their active participations in these research findings. Secondly, we are grateful to the financial supporter; Global IT Consultancy for Innovations without whom the current research work could not be carried out. Finally, we pay my special tribute to the great artist; Leonardo De Vinci for his splendid pieces of art.

\section{References}

Adair, J. (2010). Decision Making and Problem Solving Strategies. London: KoganPage Press.

Ahmad, G. (2011). Cognitive Impact of Eye Movements in Picture Viewing. International Journal of Intelligent Information Processing, 2(1), 1-8. http://dx.doi.org/10.4156/ijiip.vol2.issue1.1

Ahmad, G. (2013). Analogical Thoughts Persuade Eye Movements during Scene Viewing. International Journal of Intelligent Information Processing, 4(4), 39-45.

Ahmad, G. (2013). Analogy Influences Eye Movements during Scene Viewing. Proceedings of International Conference on Computing and Convergence Technology, Republic of Korea, (pp. 1-4). 
Ahmad, G. (2014). Analogical Thinking Induces Eye Movements in Scene Viewing. International Journal of Machine Learning and Computing, 4(1), 94-98. http://dx.doi.org/10.7763/ijmlc.2014.v4.393

Ahmad, G. (2014). Dynamics of Eye Gazing rely on Associative Relevance in Scene Viewing. Journal of Convergence Information Technology, 9(2), 35-42.

Ahmad, G. (2014). Eye Movements Look for Analogical Patterns during Scene Viewing. Journal of Economics, Business and Management, 2(4), 285-288.

Ahmad, G. (2014). Flow of Analogical Thoughts Controls Eye Movements in Scene Viewing. Journal of Next Generation Information Technology, 5(4), 118-125.

Ahmad, G. (2015). Associative Relevance Based Stimulus Shifts Focus in Eye Movements. International Business Research, 8(10), 25-34. http://dx.doi.org/10.5539/ibr.v8n10p25

Ahmad, G. (2015). Dynamic Shifts in Visual Focus of Eye Movements Emphasize Associative Relevancy. Research in World Economy, 6(4), 18-28. http://dx.doi.org/10.5430/rwe.v6n4p18

Ahmad, G. (2015). Eye Fixation curves along Analogical Thinking in Scene Viewing. International Journal of Engineering and Industries, 6(1), 54-62.

Ahmad, G. (2015). Visual Focus of Attention Actively Associates Relevancy in Eye Movements. Journal of Business Theory and Practice, 3(2), 209-223.

Bird, G. D., Lauwereyns, J., \& Crawford, M. T. (2012). The role of eye movements in decision making and the prospect of exposure effects. Vision Research, 60, 16-21. http://dx.doi.org/10.1016/j.visres.2012.02.014

Bly, B. M., \& Rumelhart, D. E. (Eds.). (1999). Cognitive Science. USA: Academic Press.

Bojko, A. (2013). Eye Tracking, the User Experience: a practical guide to research. New York: Rosenfeld Media.

Brown, T. (1999). The Science and Art of Tracking. New York: NY: Berkley Books.

Duchowski, A. (2003). Eye Tracking Methodology: Theory and Practice. London: Springer Verlag. http://dx.doi.org/10.1007/978-1-4471-3750-4

Gentner, D. (1983). Structure-mapping: A theoretical framework for analogy. Cognitive Science, 7, 155-170. http://dx.doi.org/10.1207/s15516709 $\operatorname{cog} 0702 \_3$

Gentner, D., \& Markman, A. B. (1997). Structure-mapping in analogy and similarity. American Psychologist, 52, 45-56. http://dx.doi.org/10.1037/0003-066X.52.1.45

Gentner, D., \& Medina, J. (1998). Similarity and the development of rules. Cognition, 65, 263-297. http://dx.doi.org/10.1016/S0010-0277(98)00002-X

Gentner, D., Rattermann, M. J., \& Forbus, K. D. (1993). The roles of similarity in transfer: Separating retrievability from inferential soundness. Cognitive Psychology, 25, 524-575. http://dx.doi.org/10.1006/cogp.1993.1013

Gompel, R. P., Fischer, M. H., Murray, W. S., \& Hill, R. L. (Eds.). (2007). Eye Movements: A Window on Mind and Brain. UK: Elsevier.

Granka, L. A., Joachims, T., \& Gay, G. (2004). Eye-Tracking Analysis of User Behavior in WWW Search. SIGIR 04. Sheffield, South Yorkshire, UK: ACM. http://dx.doi.org/10.1145/1008992.1009079

Griffin, Z. M. (2004). Why look? Reasons for eye movements related to language production. In H. A. Ferreira (Ed.), The integration of language, vision, and action: Eye movements and the visual world (pp. 213-247). New York: Psychology Press.

Henderson, J. M., \& Hollingworth, A. (1998). Eye movements during Scene Viewing. An Overview. In G. W. Underwood (Ed.), Eye Guidance in Reading and Scene Perception (pp. 269-293). Oxford: Elsevier. http://dx.doi.org/10.1016/B978-008043361-5/50013-4

Henderson, J. M., \& Hollingworth, A. (1999). High-level Scene Perception. Annual Review of Psychology, 50(1), 243-271. http://dx.doi.org/10.1146/annurev.psych.50.1.243

Hermansen, O. (2015). Implementing eye movements in business applications. Brage Bibsys, Hogskolen i Ostfold.

Hoffman, J. E. (1998). Visual Attention and Eye Movements. In H. Pashler (Ed.), Attention (pp. 119-153). London: Psychology Press. 
Hofstadter, D., \& Sander, E. (2013). Surfaces and Essences: Analogy as the Fuel and Fire of Thinking. New York: Basic Books.

Holsanova, J. (2008). Discourse, Vision, and Cognition. New York: John Benjamins Publishing Company. http://dx.doi.org/10.1075/hcp.23

Koller, M., Salzberger, T., Brenner, G., \& Walla, P. (2012). Broadening the range of applications of eye-tracking in business research. Analise, Porto Alegre, 23(1), 71-77.

Liversedge, S. P., Gilchrist, I. D., \& Everling, S. (2011). The Oxford Handbook of Eye Movements. Oxford: Oxford University Press. http://dx.doi.org/10.1093/oxfordhb/9780199539789.001.0001

Nikolaev, A. R., Pannasch, S., Ito, U., \& Belopolsky, A. (Eds.). (2014). Eye movement-related brain activity during perceptual and cognitive processing. Frontiers Research Topics. Frontiers E-books. http://dx.doi.org/10.3389/978-2-88919-273-1

Orquin, J. L., \& Loose, S. M. (2013). Attention and choice: A review on eye movements in decision making. Acta Psychologica, 144(1), 190-206. http://dx.doi.org/10.1016/j.actpsy.2013.06.003

Pan, B., Hembrooke, H. A., Gay, G. K., Granka, L. A., Feusner, M. K., \& Newman, J. K. (2004). The Determinants of Web Page Viewing Behavior: An Eye-Tracking Study. Association for Computing Machinery, 147-154. http://dx.doi.org/10.1145/968363.968391

Pan, B., Hembrooke, H., Joachims, T., Lorigo, L., Gay, G., \& Granka, L. (2007). In Google We Trust: Users' Decisions on Rank, Position, and Relevance. Journal of Computer-Mediated Communication, 12, 801-823. http://dx.doi.org/10.1111/j.1083-6101.2007.00351.x

Rayner, K. (1992). Eye movements and visual cognition: scene perception and reading. New York: Springer Verlag. http://dx.doi.org/10.1007/978-1-4612-2852-3

Snowden, R., Thompson, P., \& Troscianko, T. (2012). Basic Vision: An introduction to visual perception. Oxford University Press.

Solso, R. L. (1994). Cognition and Visual Arts. London: MIT Press.

Solso, R. L. (2003). The psychology of art and the evolution of the conscious brain. London: MIT Press.

Stark, L., \& Ellis, S. R. (1981). Scanpaths Revisited: Cognitive Models Direct Active Looking. In R. A. Dennis \& F. Fisher (Eds.), Eye Movements: Cognition and Visual Perception (pp. 193-226). Hillsdale: Lawrence Erlbaum Associates.

Tommasi, L., Peterson, M. A., \& Nadel, L. (Eds.). (2009). Cognitive Biology: Evolutionary and Developmental Perspectives on Mind, Brain and Behavior. London: MIT Press. http://dx.doi.org/10.7551/mitpress/9780262012935.001.0001

Tsotsos, J. K. (2011). A Computational Perspective on Visual Attention. London: MIT Press. http://dx.doi.org/10.7551/mitpress/9780262015417.001.0001

Turner, M. (2006). The Artful Mind-Cognitive Science and the riddle of human creativity. Oxford: Oxford University Press. http://dx.doi.org/10.1093/acprof:oso/9780195306361.001.0001

VanRullen, R., \& Thorpe, S. J. (2001). The time course of visual processing: from early perception to decision-making. Journal of Cognitive Neuroscience, 13(4), 454-461. http://dx.doi.org/10.1162/08989290152001880

Viegas, F. B., \& Wattenberg, M. (2007). Artistic Data Virtualization: Beyond Visual Analytics. Second International Conference on Online Communities and Social Computing (pp. 182-191). http://dx.doi.org/10.1007/978-3-540-73257-0_21

Wells, A. (2000). Emotional Disorders and Metacognition: Innovative Cognitive Therapy. West Sussex: John Wiley \& Sons. http://dx.doi.org/10.1002/9780470713662

\section{Copyrights}

Copyright for this article is retained by the author(s), with first publication rights granted to the journal.

This is an open-access article distributed under the terms and conditions of the Creative Commons Attribution license (http://creativecommons.org/licenses/by/3.0/). 